\title{
Influence of annealing in vacuum and in air on magnetic, crystallographic and morphological properties of thin YIG films
}

\author{
A. Siblini ${ }^{1}$, I. Khalil ${ }^{1}$, J.P. Chatelon ${ }^{1}$, M.F. Blanc-Mignon ${ }^{1}$, D. Jamon ${ }^{1}$ and J.J. Rousseau ${ }^{1}$ \\ ${ }^{1}$ Université de Lyon, F-42023, Saint Etienne, France; Université de Saint Etienne, Jean Monnet, F-42023, Saint Etienne, \\ France; LT2C, F-42023, Saint Etienne, France.
}

\begin{abstract}
The Yttrium Iron Garnet (YIG) is chosen by our laboratory for the integration of passive components in the microwaves domain and for the miniaturization of integrated inductors and transformers. Magnetic and morphological characterizations are essential for the development of these components. To fabricate a micro-inductor of solenoid type, we have deposited on an alumina substrate, by RF sputtering, a thin YIG film between two layers of copper. We have also used the photolithography technique in a clean room to obtain the desired pattern of the coil. The YIG films are amorphous after deposition, the annealing at $740^{\circ} \mathrm{C}$ for 2 hours is necessary for them to be crystallized and to have magnetic properties. To avoid the deterioration of copper layers, the Classical Thermal Annealing (CTA) was replaced by a Vacuum Thermal Annealing (VTA). Before manufacturing the integrated inductor, it is interesting to do the magnetic, crystallographic and morphological characterizations of YIG films after annealing with both techniques of thermal treatment. To check the quality of the prototype, we have done different characterizations: VSM, Kerr effect, XRD, SEM. The results obtained with VTA were better than of CTA comparing them with bulk YIG properties.
\end{abstract}

\section{Introduction}

The main purpose of this work is to study the influence of annealing in vacuum and in air on magnetic, crystallographic and morphological properties of YIG thin films. The characterization of these films is of great interests in magneto-optics, microwaves and radiofrequency domains. They are used in our Laboratory for the miniaturization of magnetic devices as planar inductors and transformers [1-3] and also for the integration of microwaves passive components like resonators, isolators and circulators [4-6].

To manufacture a planar micro-inductor of solenoid type, a YIG thin film is deposited on an alumina substrate, between two copper layers, by RF sputtering technique. A post thermal annealing at $740^{\circ} \mathrm{C}$ for 2 hours is necessary to make the YIG film crystallize and to obtain satisfactory magnetic properties [7].

The manufacturing techniques of magnetic layers are well controlled in our Laboratory; we have published several articles on these techniques [8-10]. We focus here, particularly on the comparison of results obtained with the two annealing techniques (CTA and VTA) to validate the VTA one. For this, we used different characterization systems as Vibrating Sample Magnetometer (VSM), Kerr effect, X-ray Diffraction Patterns (XRD), profilometer and Scanning Electron Microscope (SEM), for the two annealing techniques.

\section{Experimental}

YIG and $\mathrm{Cu}$ films are deposited on alumina substrate using a radio-frequency magnetron non-reactive sputtering system. To obtain films of good qualities, all conditions and parameters concerning the background pressure, purity and flow rate of argon, the distance between the target and the substrate, the radio-frequency power of the magnetron cathode etc..., are specified in previous works [11-13].

Two kinds of post-deposition annealing are employed in air or vacuum: CTA and VTA respectively. The samples are heated during 2 hours at $740^{\circ} \mathrm{C}$. The temperature is regulated using a PID controller. The heating and cooling times ratios are fixed to $6^{\circ} \mathrm{C} / \mathrm{min}$. For CTA, we used a conventional furnace, having four halogen bulbs of $450 \mathrm{~W}$ each, but for VTA, we have built a special furnace capable to work in background pressure of about $10^{-3} \mathrm{~Pa}$ [9]. It contains two heating spiral resistors introduced inside two hollow cylinders of copper. The sample is put between the heaters (figure 1). As the YIG film is deposited between two copper layers, we have to make VTA to avoid the deterioration of the first layer (the second one is deposited after annealing).

The geometrical shape of copper windings is obtained by photolithography technique in a clean-room. A 
profilometer is used to check the homogeneity of thickness. The SEM images are analyzed to characterize the morphological properties of YIG and $\mathrm{Cu}$ films. XRD patterns and magnetic hysteresis loops obtained by VSM and Kerr effect to see if the YIG films are wellcrystallized magnetic material.

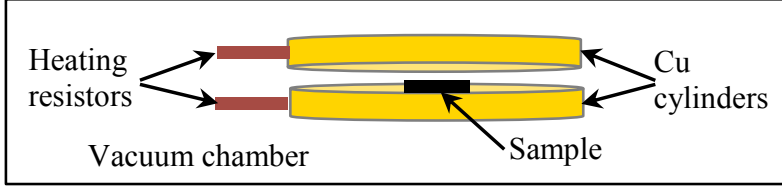

Fig. 1. Furnace used for vacuum annealing.

\section{Results}

\subsection{Magnetic properties}

The hysteresis loops are measured by VSM and Kerr effect, VSM measurements were performed with magnetic field perpendicular (p-plane) and parallel (inplane) to the YIG film. The hysteresis loops of Figure 2 (a) and (b) are performed for YIG films, having a thickness of $2.4 \mu \mathrm{m}$, deposited on an alumina substrate With VTA and CTA thermal treatment respectively.

One can see that in the 2 cases the YIG films have very close magnetic characteristics for Kerr effect and VSM p-plane. The shape of the hysteresis loops proves that YIG films have soft magnetic properties, which correspond to a low remanent magnetization. YIG films exhibit a strong anisotropy of form to reach saturation magnetization (165 mT for VTA and $145 \mathrm{mT}$ for CTA), the saturation magnetization for VTA is very closed to the bulk YIG one (175 mT) [14].

\subsection{Crystallographic properties}

Figure 3 shows the XRD patterns of YIG films deposited on alumina substrate and annealed at $740{ }^{\circ} \mathrm{C}$ for 2 hours by the two processes: CTA and VTA. The crystallization is complete and corresponds to the YIG phase that is indexed with JCPDS 43-0507. The alumina substrate is referenced with JCPDS 46-1212. The diffraction peaks corresponding to CTA and VTA show any significant difference on the crystallize properties of the YIG films. But the shape of these peaks has permitted us to estimate the grain size of about $140 \mathrm{~nm}$ for VTA and $180 \mathrm{~nm}$ for CTA by using Scherer formula.

\subsection{Morphological properties}

As the thermal expansion coefficients of the substrate and the deposited films are very different, the stress during the thermal annealing produces cracks formation or detachment of these layers. For this reason we have made
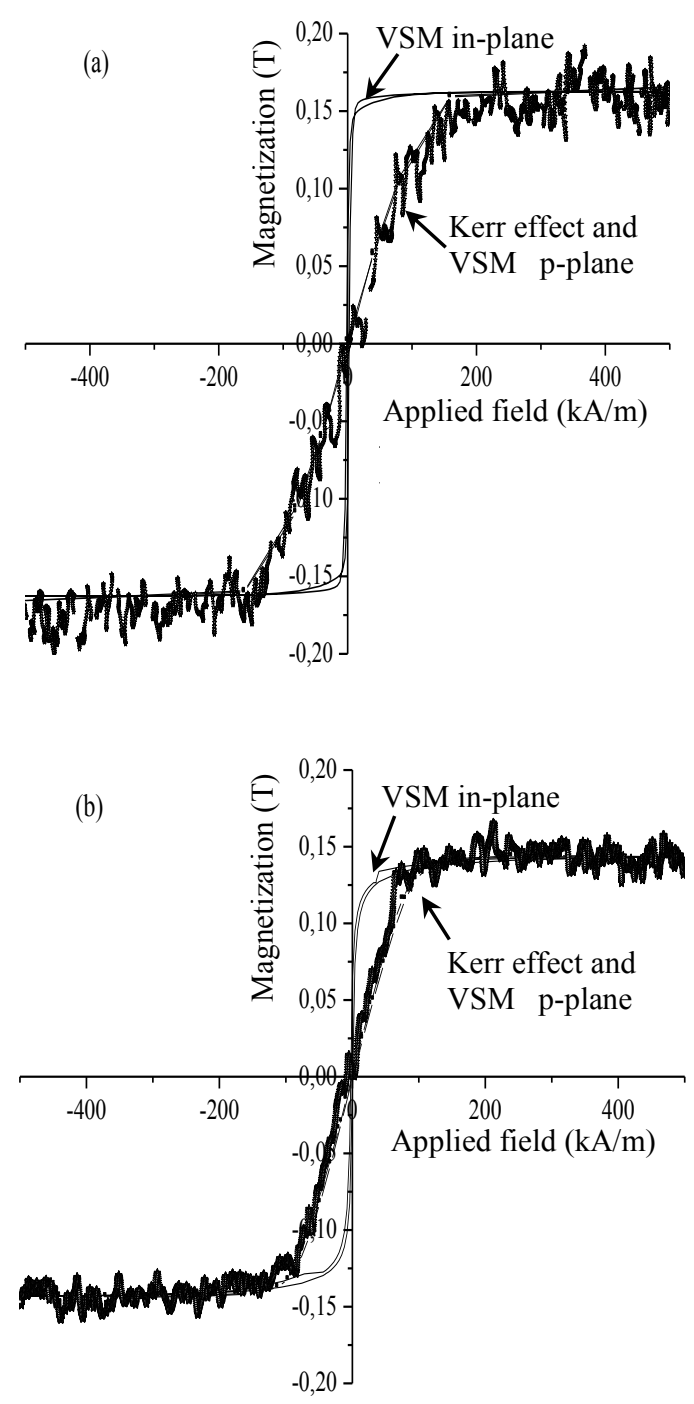

Fig. 2. Magnetic hysteresis loops of YIG thin film deposited on alumina substrates (a) VTA and (b) CTA.

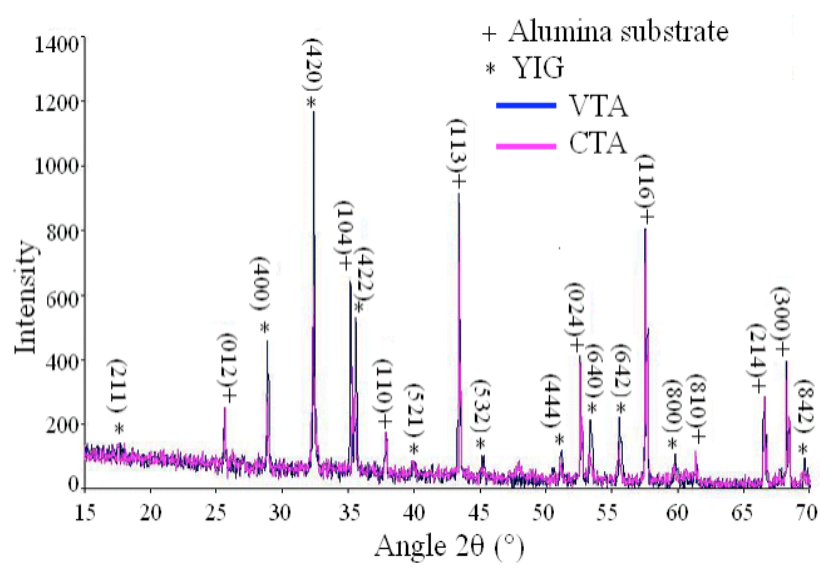

Fig. 3. X-ray diffraction patterns of YIG films deposited on alumina substrates and annealed at $740^{\circ} \mathrm{C}$ during 2 hours: (a) for VTA and(b) for CTA. 
different tests by varying the parameters of the annealing mode and the substrate roughness $(\rho)$. The annealing of YIG thin film is properly controlled in our laboratory [6, 13, 11 and 15]. The sample is annealed during 2 hours at $740{ }^{\circ} \mathrm{C}$. The heating and cooling rates are very important for the morphology of YIG films. Heating and cooling rates of $6^{\circ} \mathrm{C} / \mathrm{min}$ have given a good surface state. To decrease the roughness of the substrate, we have used a polishing machine. Different tests have permitted to find the better morphological state for $\rho \cong 90 \mathrm{~nm}$ [9-10].

Figures 4 (a) and (b) show 2 SEM images of YIG thin films having a thickness of $2.4 \mu \mathrm{m}$ and deposited on alumina substrate for the 2 kinds of heat treatment: VTA and CTA respectively. One can see that there are no visible cracks or detachments. We notice also that the surface corresponding to VTA is smoother than the CTA one. This result is in concordance with that of the grain size previously obtained in the 3.1 section. By using a profilometer, we have noticed a uniform cartography of thickness for VTA and CTA.
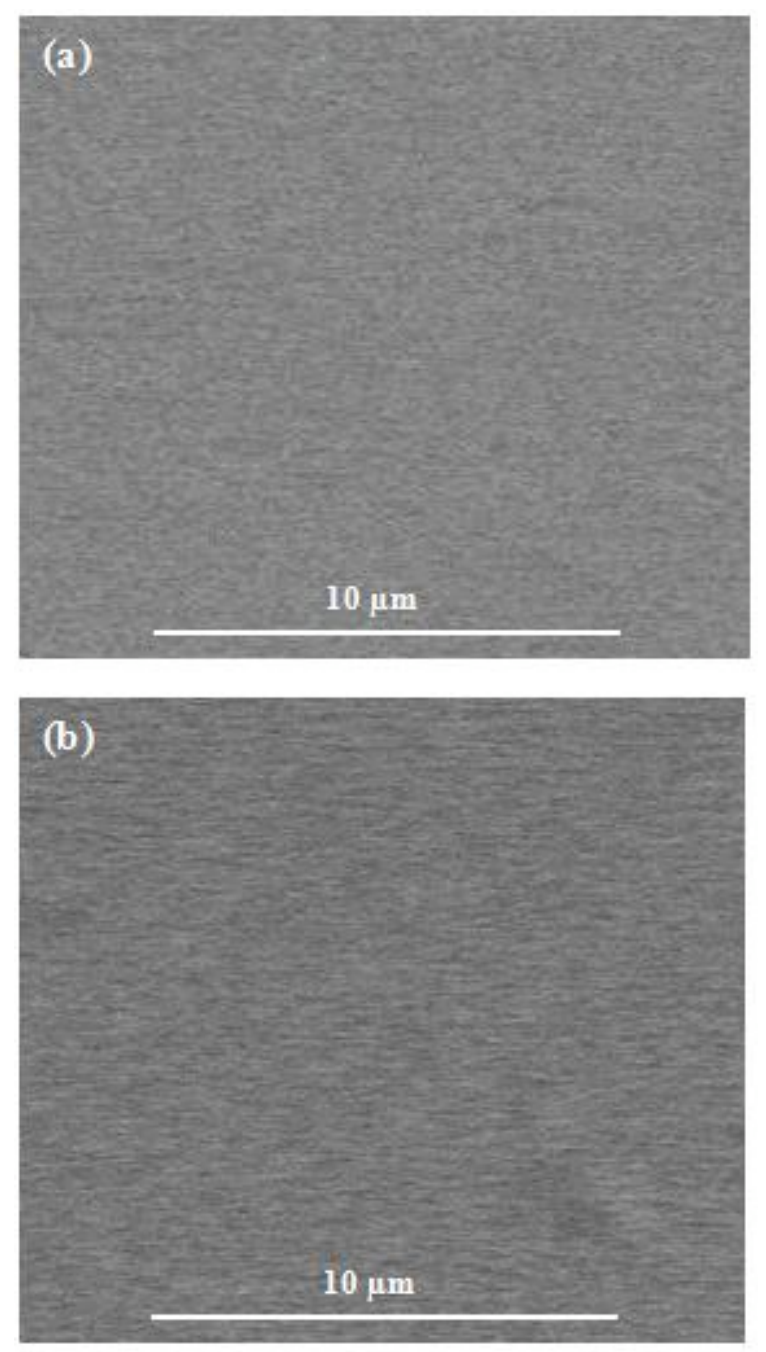

Fig. 4. SEM images (same magnification) of YIG thin films deposited on alumina substrates for the two cases: (a) VTA and (b) CTA

\section{Conclusion}

Thin and thick layers of YIG have been used in our Laboratory to study different integrated passive components [3-6]. While studying these devices, we have encountered several mechanical and electrical problems: cracks, detachment of YIG or $\mathrm{Cu}$ films from the substrate, deterioration of $\mathrm{Cu}$ films, open or short circuit. The alumina substrate roughness and thermal annealing mode play an important role on the morphological, electrical and magnetic properties of these films. All these problems have been encountered by varying different parameters; we have established a protocol permitting to manufacture a prototype of good quality [911]. The VTA mode is not used in the case that the conducting winding is deposited after the YIG film. But for the inductors of solenoid type we have to deposit the first layer of copper before the YIG film, in this case, we have to use the VTA mode to avoid the deterioration of copper layer. This work is essentially dedicated to the comparison of results obtained by the 2 modes: VTA and CTA. The magnetic, crystallographic and morphological characterizations have given better results for the VTA mode. The hysteresis loops show that YIG films have soft magnetic properties for the two modes. But, the saturation magnetization of YIG film for VTA is closer to the YIG bulk than the CTA one and the anisotropy of form is more marked for VTA that is important for the high frequency applications. The XRD for CTA and VTA show the crystallographic and magnetic properties of the YIG films (JCPDS 43-0507 YIG reference). The grain size calculated from Scherer formula gives smaller size for VTA. And finally, the SEM images have shown the good morphological states for the 2 modes of heat treatment; however the surface of YIG film for VTA is less rough than the CTA one.

\section{References}

1. J. S. Song, B. K. Min, D. Y. Jeong, H. S. Kim, J. S. Heo, J. Magn. Magn. Mater. 234, 494 (2001).

2. M. Yamaguchi, Y. Miyazawa, K. Kaminishi, H. Kikouchi, S. Yabukami, K. I. Arai, T. Suzuki, J. Magn. Magn. Mater. 268, 170 (2004).

3. E. Gamet, J. P. Chatelon, T. Rouiller, B. Bayard, G. Noyel, J. J. Rousseau, J. Magn. Magn. Mater. 288, 121 (2005).

4. D. Vincent, T. Rouiller, C. R. Simovski, B Bayard, and G. Noyel, IEEE Trans. Microwave Theory Tech. 53, 1174 (2005).

5. B. Bayard, D. Vincent, C. R. Simovski and G. Noyel, IEEE Trans. Microwave Theory Tech. 51, 1809 (2003).

6. S. Capraro, T. Boudiar, T. Rouiller, J. P. Chatelon, B. Bayard, M. Le-Berre, B. Payet-Gervy, M. F. Blanc-Mignon and J. J. Rousseau, Microwave and Optical Tech. Let. 42, 470 (2004).

7. S. H. Gee, Y. K. Hong, D. W. Erickson, T. Tanaka, M. H. Park, J. Appl. Phys. 93, 7507 (2003). 
8. A. Kriga D. Allassem, M. Soultan, A. Siblini, J.P. Chatelon, B. Allard and J.J. Rousseau, J. Magn. Magn. Mater. 324, 14, 2227, (2012)

9. A. Siblini, I. Khalil and J.P. Chatelon, sensor Letters 5, 51 (2007)

10. A. Siblini, I. Kalil, J. P. Chatelon and j.j. Rousseau Advanced Materials Research , 324, 290 (2011)

11. B. Abdel Samad, M. -F. Blanc-mignon, M. Roumie, A.Siblini, J. P. Chatelon, M. Korek, Eur. Phys. J. Appl. Phys., 50, 10502 (2010).

12. S. Capraro, J.P. Chatelon, M. Le Berre, H. Joisten, T. Rouiller, B. Bayard, D. Barbier and J.J. Rousseau, J. Magn. Magn. Mater., 272-276, 1805 (2004)

13. T. Boudiar, S. Capraro, T. Rouiller, M. F. BlancMignon, B. Payet-Gervy, M. Le Berre, J.J. Rousseau, Phys. Stat. Sol. (c) 1, 3347 (2004).

14. N. B. Ibrahim, C. Edwards, S. B. Palmer, J. Magn. Magn.Mater. 200, 194 (2000).

15. T. Boudiar, B. Payet Gervy, M. F. Blanc Mignon, J. J. Rousseau, M. Le Berre, H. Joisten, J. Magn. Magn. Mater. 284, 77 (2004). 